\title{
Gesetzliche Rente: Bedeutet Teilhabeäquivalenz Verteilungsneutralität? - Ein weiterer Irrtum
}

\author{
Ein Grundprinzip der Gesetzlichen Rentenversicherung in Deutschland ist das der \\ „Teilhabeäquivalenz“, das die Proportionalität zwischen Rentenansprüchen und \\ Beitragszahlungen ausdrückt. Verfechter des Systems rechtfertigen dieses Prinzip mit dem \\ Ziel der Verteilungsneutralität. Gerade diese ist jedoch verletzt, weil sich klar definierbare \\ Bevölkerungsgruppen in ihrer Lebenserwartung unterscheiden.
}

Die Berechnung der gesetzlichen Rente erfolgt nach dem Prinzip der Teilhabeäquivalenz, bisweilen auch „Beitragsäquivalenz" genannt. Es wird in der juristischen Literatur interessanterweise mit zwei unterschiedlichen Zielsetzungen begründet:

- zum einen mit dem Ziel der (intragenerativen) Verteilungsneutralität: es drücke aus, dass innerhalb jeder Alterskohorte gleiche Beitragszahlungen zu gleichen Rentenansprüchen führten;

- zum anderen mit dem Ziel, die Stellung des Versicherten in der Einkommenshierarchie im Alter aufrechtzuerhalten. $^{2}$

Beide Ziele stehen im Widerspruch zueinander, von Beitragsäquivalenz kann in Wahrheit keine Rede sein, und sogar die Verteilungsneutralität ist gröblich verletzt. Die in den Zielen implizit enthaltenen Behauptungen übersehen nämlich die zeitliche Dimension sowohl der Beitragszahlung als auch des Rentenbezugs.

\section{Zeitliche Dimension}

Die Aufrechterhaltung der Position in der Einkommenshierarchie verlangt, dass sich der (monatliche) Rentenanspruch allein am durchschnittlichen Einkommen des Versicherten während seiner Erwerbsphase, gemessen am mittleren Einkommen aller Versicherten, orientiert. Die Einzahlungsdauer dürfte dagegen keine Rolle spie-

Der Titel bezieht sich auf den Aufsatz von J. Ragnitz, W. Nagl: Drei Irrtümer über die gesetzliche Rente, in: Wirtschaftsdienst, 92. Jg. (2012), H. 11, S. 759-762.

1 Vgl. F. Ruland (Hrsg.): Handbuch der gesetzlichen Rentenversicherung, Neuwied, Frankfurt a.M. 1990, Kapitel 19, Rz. 37.

2 So etwa A. Wallrabenstein: Versicherung im Sozialstaat, Tübingen 2009, S. $196 \mathrm{f}$ len. Bezieht man diese jedoch in die Rentenberechnung ein und ist sie unterschiedlich lang, so kommt es natürlich vor, dass Versicherte mit mehr Versicherungsjahren andere mit weniger Jahren im Alter in der Einkommenshierarchie überholen. Dann wird das zweite Ziel zu Gunsten des ersten geopfert.

Individuelle Rentenansprüche richten sich zudem ausschließlich nach den erworbenen Entgeltpunkten, und diese sind als Proportion des (beitragspflichtigen) Einkommens des Versicherten zum Durchschnittseinkommen aller Versicherten in der jeweiligen Periode definiert. Die tatsächliche Beitragshöhe spielt gar keine Rolle. Dies kann zu erheblichen Verstößen gegen die „Beitragsäquivalenz" führen, wenn zwei Versicherte aus einer Kohorte immer gleich viel verdient, aber in unterschiedlichen Jahren (etwa wegen Kindererziehung), in denen der Beitragssatz sich verändert hat, ausgesetzt haben: eine Arbeitnehmerin mit 5000 Euro Monatseinkommen, die 2012 gearbeitet hat und 2013 aussetzt, hat dadurch 420 Euro mehr Beiträge gezahlt als ihre Kollegin mit gleichem Verdienst, die 2012 ausgesetzt hat und 2013 arbeitet. Die Rentenansprüche sind dennoch gleich groß.

Prof. Dr. Friedrich Breyer lehrt Volkswirtschaftslehre an der Universität Konstanz. Er ist derzeit Vorsitzender der Deutschen Gesellschaft für Gesundheitsökonomie. 


\section{Unterschiedliche Lebenserwartung}

Noch gravierender als die Beitragssatzschwankungen ist jedoch die Tatsache, dass zwar die Dauer der Versicherungszeit in die Berechnung der Rentenansprüche einbezogen wird, nicht jedoch die erwartete Rentenlaufzeit. Damit werden zwei inkommensurable Größen in Beziehung gesetzt: Die über das Erwerbsleben kumulierten Entgeltpunkte und der monatliche Rentenanspruch. Diese Inkonsistenz wäre unkritisch, wenn die erwartete Rentenlaufzeit bei allen Versicherten die gleiche wäre, alle Unterschiede zwischen den Laufzeiten also zufällig wären und es keine systematischen Zusammenhänge mit anderen soziodemographischen Merkmalen gäbe. Ansonsten ist sie jedoch problematisch, wenn es klar abgrenzbare Gruppen in der Bevölkerung gibt, für die zwei Eigenschaften erfüllt sind:

1. ihre statistische Lebenserwartung liegt deutlich unter derjenigen in der Gesamtbevölkerung, so dass 1 Euro ihres Beitrags weniger wert ist als der anderer Versicherter, und

2. es gilt als gesellschaftlich unerwünscht, diese Gruppen gegenüber dem Rest der Gesellschaft zu benachteiligen.

Eine bekannte Tatsache ist die geringere Lebenserwartung der Männer im Vergleich zu den Frauen. Hier ist die erste Eigenschaft erfüllt, Eigenschaft 2 jedoch nicht. Erst kürzlich hat der Gesetzgeber durchgesetzt, dass das Geschlecht bei privaten Versicherungsverträgen kein Merkmal bei der Prämienberechnung sein darf („Unisex-Tarife"). Dies benachteiligt Männer bei Leibrenten-Verträgen, begünstigt sie jedoch in der Lebensversicherung. Was in der Privatversicherung Gesetz ist, muss erst recht in der Sozialversicherung gelten: Die Gesetzliche Rentenversicherung (GKV) verteilt Einkommensteile von Männern zu Frauen um, und es gilt als gesellschaftlicher Konsens, dass daran nicht gerüttelt wird.

Eine weitere, in zahlreichen wissenschaftlichen Untersuchungen erhärtete Tatsache ist, dass die statistische Lebenserwartung positiv mit dem Einkommen korreliert. In der deutschen Rentenversicherung bedeutet ein zusätzlicher Entgeltpunkt pro Jahr bei Männern einen Zuwachs an Lebenserwartung um vier Jahre. ${ }^{3}$ Für das Merkmal "geringes Arbeitseinkommen“ ist also die erste Eigenschaft erfüllt, es handelt sich ferner um abgrenzbare

3 Vgl. F. Breyer, S. Hupfeld: Fairness of Public Pensions and Old-Age Poverty, in: FinanzArchiv, 65. Jg. (2009), S. 358-380; sowie für Frauen: F. Breyer, J. Marcus: Income and Longevity Revisited: Do High-Earning Women Live Longer?, DIW Discussion Paper, Nr. 1037, Juli 2010.
Abbildung 1

Lineare und degressive Zuteilung von

Entgeltpunkten

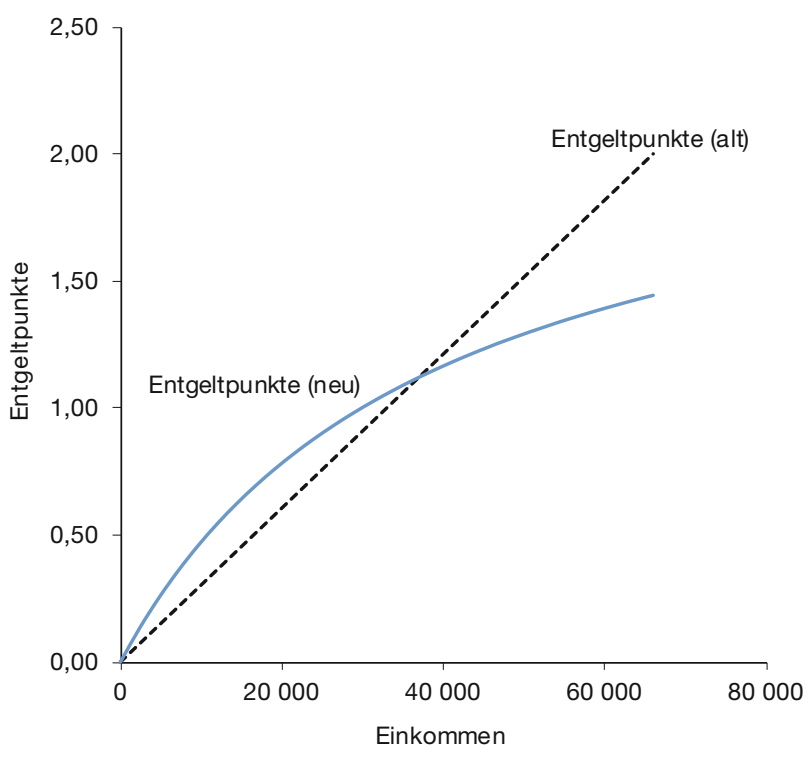

Gruppen, denn das (beitragspflichtige) Einkommen wird von der GRV jedes Jahr erfasst, und Eigenschaft 2 ist ebenfalls erfüllt, da sich alle politischen Lager gegenwärtig bemühen, Regelungen zu finden, um Geringverdiener im Alterseinkommen besser zu stellen.

\section{Ein Vorschlag}

Technisch ließe sich die Berücksichtigung der Lebenserwartung dadurch lösen, dass von einem bestimmten Stichtag an die Zuteilung der jährlichen Entgeltpunkte bezogen auf das beitragspflichtige Einkommen nicht mehr einer linearen, sondern einer degressiven Formel folgt, die den Effekt der mit dem Einkommen steigenden Rentenlaufzeit kompensiert. Vergleiche dazu Abbildung 1, in der die gestrichelte Linie die derzeit gültige Vergabe der Entgeltpunkte darstellt und die durchgezogene Linie die verteilungsneutrale Vergabe unter Berücksichtigung der einkommensspezifischen Lebenserwartung. Eine solche Umstellung hätte zur Folge, dass in einem Übergangszeitraum von ca. 50 Jahren die Altersrenten der Neurentner mehr und mehr die erwartete Laufzeit berücksichtigen. Durch die damit verbundene Anhebung der Renten von Geringverdienern würde sich nach und nach auch das Problem der Altersarmut entschärfen.

Gegen eine derartige Änderung der Rentenformel wird eine Reihe von Einwänden vorgebracht, die jedoch durchweg entkräftet werden können: 
1. „Die Berücksichtigung der erwarteten Rentenlaufzeit ist systemfremd und wäre ein Novum in der GRV." Diese Behauptung ist falsch, denn die schon heute geltenden Abschläge bei vorzeitigem Renteneintritt sind ausschließlich dadurch begründet, dass sich durch den vorzeitigen Eintritt die Laufzeit verlängert.

2. „Die Aufnahme eines risikobezogenen Elements in die Rentenformel wäre ein Dammbruch. Sie würde sofort Forderungen weiterer Gruppen (z.B. der Raucher) nach sich ziehen, eine höhere Rente zu bekommen." Diesem Argument kann man entgegenhalten, dass es mit Ausnahme der Geringverdiener keine abgrenzbare Gruppe gibt, für die die oben genannten Eigenschaften 1 und 2 erfüllt sind. Im Übrigen sind Raucher nicht einmal eine abgrenzbare Gruppe, da die Lebenserwartung von der Menge des Zigarettenkonsums abhängt.

3. „Die Rentenversicherung versichert Menschen schon von Geburt an gegen das Risiko der Langlebigkeit. Diesen Risikoschutz würde man mit der Differenzierung aufgeben." Die analoge Behauptung trifft für die Krankenversicherung zu, aber in der Rentenversicherung überzeugt sie nicht. Erstens bleibt festzuhalten, dass das individuelle Langlebigkeitsrisiko nach wie vor zu $100 \%$ versichert bleibt. Aus dem Versicherungsschutz ausgeklammert würde lediglich die systematische, durch das Einkommen vorhersagbare Abweichung vom Durchschnitt. Zweitens ist die Langlebigkeit als solche kein Übel (wie etwa Krankheit), sondern wird von den meisten Menschen angestrebt. Drittens ist die Langlebigkeit nur zu einem geringen Teil genetisch festgelegt, zum größeren Teil jedoch durch die Lebensumstände bedingt. Es ist eine Werturteilsfrage, ob man die These akzeptiert, dass den Bevölkerungsgruppen, denen durch die Lebensumstände (oder durch eigene Bemühungen) eine höhere statistische Lebenserwartung zuteil wurde, auch zuzumuten ist, dass sie für den damit verbundenen zusätzlichen Lebensunterhalt selbst aufkommen. Falls es stimmt, dass eine höhere Lebenserwartung in der Regel auch mit einer größeren Leistungsfähigkeit im siebten Lebensjahrzehnt einhergeht, wäre es Angehörigen dieser Gruppe auch möglich, den Abschlag in der monatlichen Rente durch eine längere Lebensarbeitszeit auszugleichen. Anders ausgedrückt würde durch die degressive Bemessung der Entgeltpunkte die Nachteile der „Rente mit 67“ für die Geringverdiener, deren Leistungsfähigkeit im Alter zwischen 60 und 67 Jahren typischerweise bereits gemindert ist, zumindest teilweise kompensiert.

4. „Das Bundesverfassungsgericht hat den Eigentumscharakter der gezahlten Beiträge zur GRV in seinen Urteilen stets betont. Daher wäre jeder Eingriff in die proportionale Zumessung der Entgeltpunkte verfassungswidrig." Auch dieses Argument ist bei näherem Hinsehen nicht so stark, wie es scheint. „Die gezahlten Beiträge" sind zunächst einmal ein Gesamtbetrag. In welchen monatlichen Rentenanspruch sich dieser übersetzt, hängt offensichtlich davon ab, welche Annahmen man über die Rentenlaufzeit trifft. Das zitierte Argument unterstellt implizit, das Verfassungsgericht halte nur eine einzige Annahme für berechtigt, nämlich die, dass man a priori über die Laufzeiten nichts weiß und daher für alle Versicherten die gleiche Länge unterstellen muss. Dies ist nach den sich immer stärker verdichtenden empirischen Befunden kaum noch aufrechtzuerhalten: Die Lebenserwartung differiert nach der sozialen Schicht, wofür das Einkommen ein guter und leicht beobachtbarer Indikator ist. Dass das Bundesverfassungsgericht sich dieser Erkenntnis auf Ewigkeit verschließen wird, ist eine unbewiesene und wenig plausible Behauptung.

\section{Fazit}

Das wesentliche Argument der Befürworter des gegenwärtigen Rentenrechts lautet, die Bemessung von Beiträgen und Rentenansprüchen sei verteilungsneutral. In ihrer Allgemeinheit ist diese Aussage natürlich nicht richtig, denn es gibt zahlreiche Umverteilungselemente in der GRV. Gemeint ist „Verteilungsneutralität zwischen den Einkommensgruppen“. Genau diese Eigenschaft ist jedoch ebenfalls verletzt, wenn man einkommensspezifische Unterschiede in der Lebenserwartung berücksichtigt. Ein Pfeiler des Sozialstaats, der bislang systematisch von den Gering- zu den Besserverdienern umverteilt, ist dringend reformbedürftig.

\section{Title: Another Preconception About Retirement Insurance}

Abstract: $A$ basic principle of statutory retirement insurance in Germany is the proportionality between earnings and retirement benefits called "Teilhabeäquivalenz". Advocates of the system defend this principle, arguing that it ensures distributive neutrality. However, this assertion is wrong because the principle combines two concepts with different time dimensions: lifetime contributions and monthly retirement benefits. If clearly defined population groups differ in their life expectancy, then this very principle achieves income redistribution, in the German case from low earners to high earners. We propose a simple change in the rules which would avoid this undesirable redistribution.

JEL Classification: H55 